\title{
TEMPS DE COMPTAGE ET INDICE DE TRI EN SPECTROMETRIE HUMAINE
}

\author{
H. FROSSARD, L. ANDRIEU, R. PERLES, \\ J. FABRE, P. FERNET, G. PRAT* \\ (Manuscrit reçu le 3 avril ${ }_{1969)}$
}

\begin{abstract}
RÉSUMÉ
Une étude statistique menée sur deux groupes d'installation de spectrométrie humaine a permis de montrer que la réduction du temps de comptage de ro minutes à 2 minutes pour les examens de spectrométrie ne diminue pas de façon significative la précision statistique de ces examens. Il est proposé dans le cadre des examens de surveillance de routine de remplacer l'examen de ro minutes «trois sondes" par un examen de 2 minutes "trois sondes".
\end{abstract}

Cette méthode permet de conclure à la non-contamination du sujet examiné :

$\mathrm{I}^{\circ}$ par le calcul d'un indice de tri,

$2^{\circ}$ par l'établissement d'un spectre.

INTRODUCTION

La spectrométrie gamma humaine constitue un excellent moyen de diagnostic de la contamination interne. Mais pour que ce diagnostic soit possible, il est nécessaire d'avoir affaire à une contamination interne par radioéléments émetteurs $\gamma$ d'énergie supérieure à $200 \mathrm{keV}$. Dans tous les autres cas : radioéléments émetteurs $\gamma$ d'énergie inférieure à $200 \mathrm{keV}$ et radioéléments émetteurs $\alpha$ ou $\beta$ purs, il est nécessaire d'effectuer des examens radiochimiques pour évaluer avec une bonne sensibilité la charge corporelle.

Dans le cadre de la surveillance du personnel exposé aux rayonnements et pour détecter d'éventuelles contaminations internes par radioéléments émetteurs $\gamma$ d'énergie supérieure à $200 \mathrm{keV}$, le Service de Santé des Armées pratique des mesures de spectrométrie humaine selon une technique classique et bien codifiée (fig. I).

Le sujet est placé dans une enceinte blindée en position semi-assise, la mesure est faite à l'aide de trois sondes à scintillation d'iodure de sodium de 5 pouces $\left(5^{\prime \prime} \times 5^{\prime \prime}\right)$ (sonde supérieure à $5 \mathrm{~cm}$ du cou; sonde ventrale à $5 \mathrm{~cm}$ de l'abdomen; sonde lombaire à $2 \mathrm{~cm}$ du sacrum). L'enregistrement est fait sur un sélecteur à 400 ou 800 canaux. La durée de la mesure est de ro minutes. Dans ces conditions, on a une excellente sensibilité et le spectre obtenu est exploitable même dans les cas de très faibles contaminations. Cependant, si on ajoute au temps de comptage le temps nécessaire à l'inscription des résultats, et celui qu'il faut au sujet examiné pour entrer et sortir de l'enceinte et pour s'installer dans le fauteuil, le débit de l'installation ne peut pas dépasser quatre examens à l'heure.

* Division d'Hygiène Atomique. Centre de Recherches du Service de Santé des Armées. 3, rue du Lieutenant R. Batany - 92-CLAMART 
Ce rythme n'est pas élevé et la surveillance systématique d'un nombre élevé de travailleurs exposés aux rayonnements peut obliger les laboratoires chargés de la spectrométrie à effectuer des examens à un rythme plus grand. Comme il n'est pas possible de multiplier les installations, lourdes et onéreuses, nous avons été amenés d'une part à simplifier l'appareillage électronique et le blindage $[\mathrm{I}]$ pour pouvoir multiplier les installations et d'autre part à étudier dans quelle mesure il était possible de réduire le temps de mesure dans l'installation complète. Il faut remarquer que la durée de la mesure en spectrométrie humaine est rarement indiquée par les différents auteurs. Dans les ouvrages spécialisés $[2,3,4,5$ et 6$]$, on trouve rarement cette précision et quand elle figure, les temps de mesure varient entre 1000 minutes et is minutes. Par contre, Jeammaire et Jammet, au Symposium de Vienne de 1961 [s], préconisaient déjà l'examen de courte durée pour des sujets contaminés, l'étalonnage se faisant à partir de sujets ayant subi pour la même contamination un examen de longue durée. Plus récemment, ANDERson [8] analyse la précision de ses mesures sur le Potassium 40 pour un comptage de trois minutes.

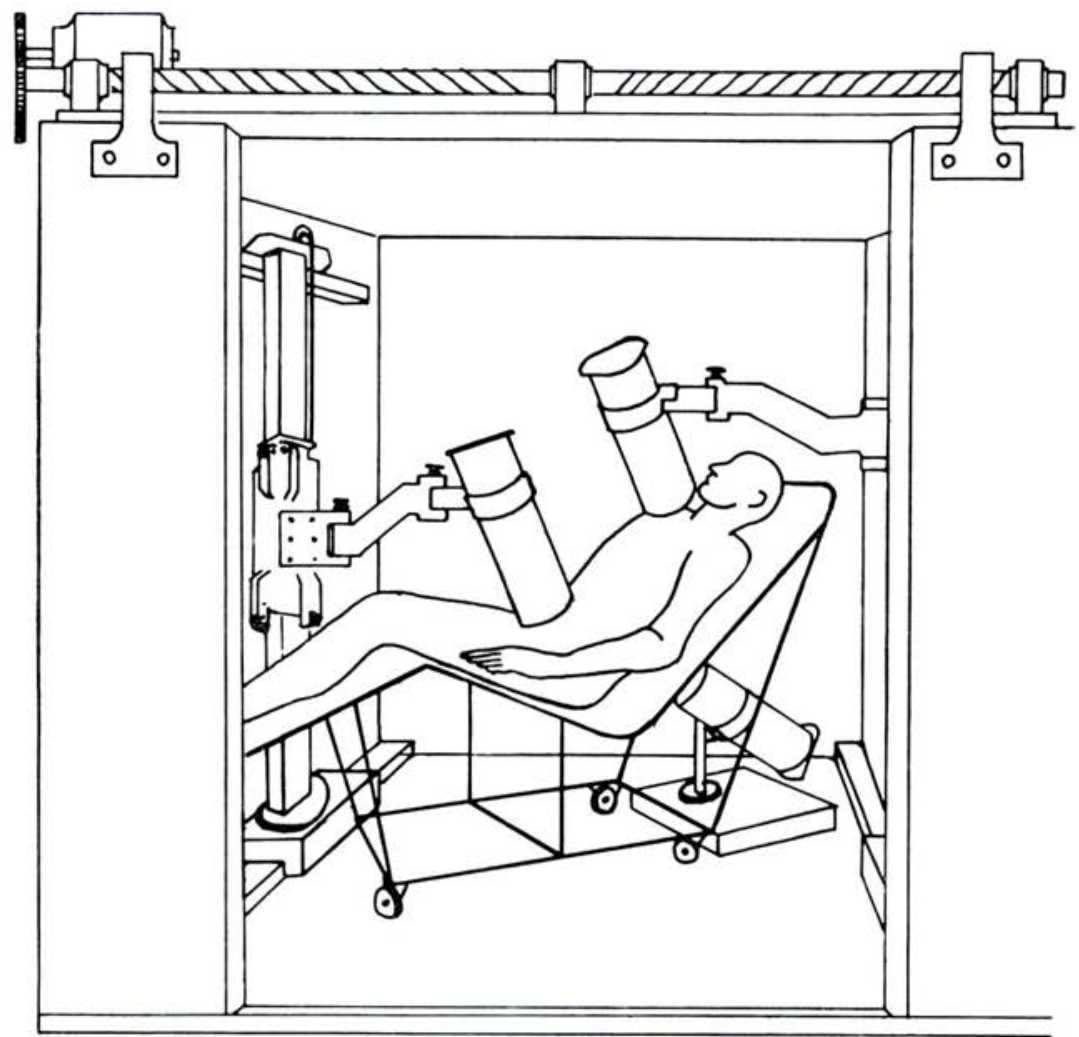

FIG. 1. - Géométrie 3 sondes utilisée en spectrométrie humaine.

Il s'agissait donc de savoir si, pour des examens de routine, on avait le droit de diminuer le temps de comptage sans altérer la signification de la mesure. Et il était nécessaire de faire une étude statistique complète.

Le but de ce travail est d'exposer l'étude faite à partir de résultats expérimentaux dans deux groupes de laboratoires de spectrométrie humaine dont nous avons la charge. Cette étude a permis de diminuer le temps de comptage, d'adopter un indice de tri et une méthode générale 
d'examen de spectrométric humaine applicable dans le cas de la surveillance de routine du personnel exposé. La validité du temps de comptage court sera étudiée après l'exposé des résultats expérimentaux. Une méthode générale d'examen sera proposée en conclusion.

\section{I - MESURES EXPÉRIMENTALES}

Deux séries de mesures ont été faites en des lieux géographiques et dans des circonstances très différentes. Les appareillages sont également différents mais pour les mesures par trois sondes la géométrie est la même dans toutes les installations qui sont au nombre de quatre.

\section{I.I. Première SÉRIE DE MESURES}

Elles ont été faites au cours de l'hiver 67-68. Il s'agit d'examens systématiques concernan 200 sujets mâles âgés de 20 à 40 ans. Les poids sont compris entre 58 et $80 \mathrm{~kg}$. Le poids moyen est de $69,5 \mathrm{~kg}$ et la taille moyenne de $\mathrm{r}, 7^{2} \mathrm{~m}$. Tous les sujets suspects de contamination ont été écartés. L'ensemble constitue donc un échantillon de population normale.

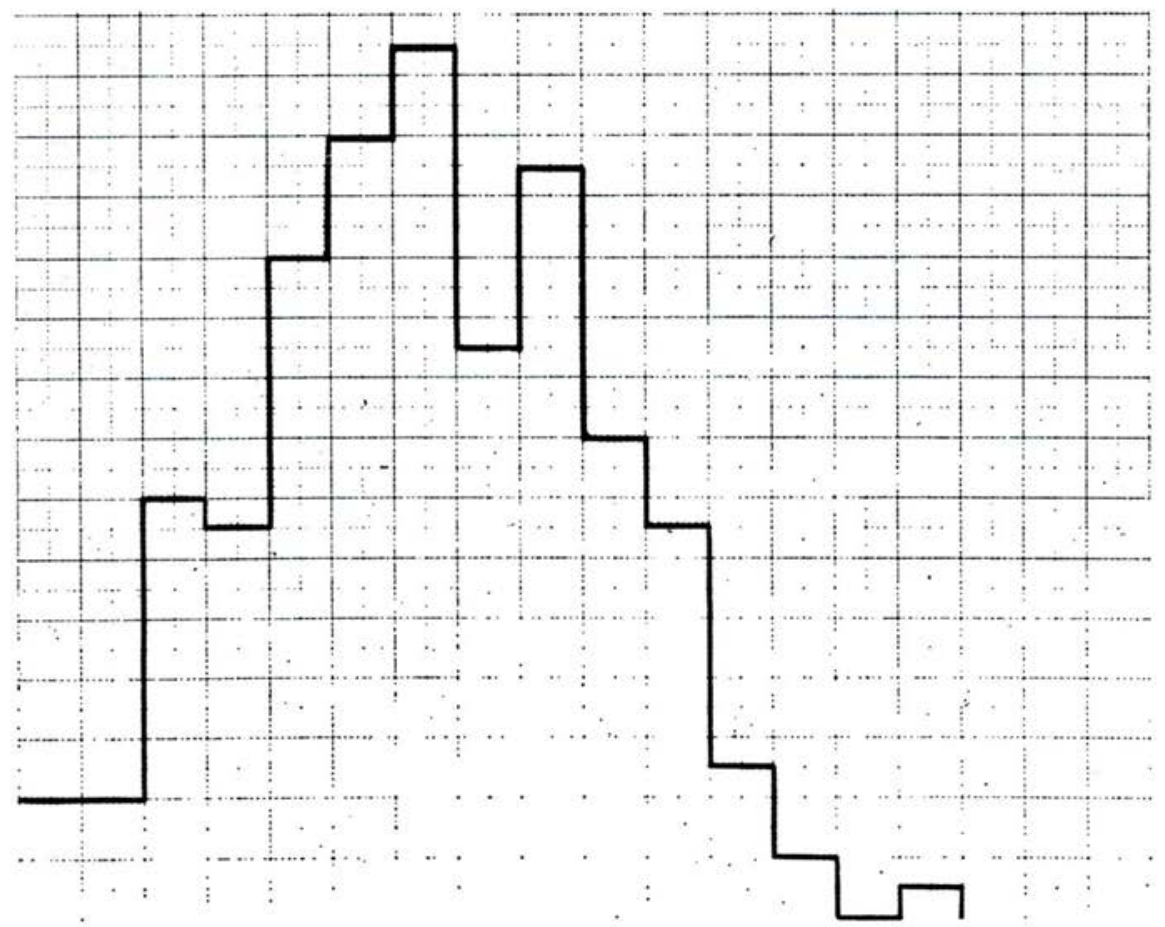

FIG. 2. - Nombre de coups par minute et par kilogramme.

Histogramme de distribution

$$
m=3,48
$$

${ }_{\text {I }}^{\text {re }}$ série de mesures (2 minutes). 
Le sujet déshabillé est pesé puis installé dans le fauteuil de l'enceinte blindée. La mesure dure II minutes et demie. Pendant 90 secondes on pratique un premier examen de courte durée dans la géométrie dite de MrLLER [9] avec une seule sonde.

Les ro minutes suivantes constituent l'examen de longue durée en géométrie trois sondes. Les deux examens sont pratiqués sur la même gamme d'énergie (o à $2 \mathrm{MeV}$ ). Pour le premier on enregistre un résultat, sur échelle de comptage, pour le second, on établit un spectre complet. Dans les deux cas on enregistre après déduction du bruit de fond le nombre total de coups.

\section{Expression des résultats}

Le poids du sujet a été retenu comme facteur de pondération et la minute comme unité de temps. Les résultats sont exprimés en coups par minute et par $\mathrm{kg}=\mathrm{CPM} / \mathrm{kg}$.

Cette expression des résultats mériterait d'être discutée mais elle est couramment pratiquée. L'activité $\gamma$ normalement détectée en spectrométrie sur un sujet normal est due au $\mathrm{Cs}^{137}$ et au $\mathrm{K}^{40}$. Ces deux éléments ont une répartition métabolique telle qu'il est parfaitement légitime de rapporter leur activité au $\mathrm{kg}$ de poids quand il n'y a pas d'autre élément contaminant.

\section{Etude statistique (fig. 2 et 3 )}

Chaque comptage donne un résultat en CPM $/ \mathrm{kg}$. L'ensemble de ces résultats constitue un échantillon dont on calcule les paramètres de distribution en faisant l'hypothèse d'une distribution gaussienne.

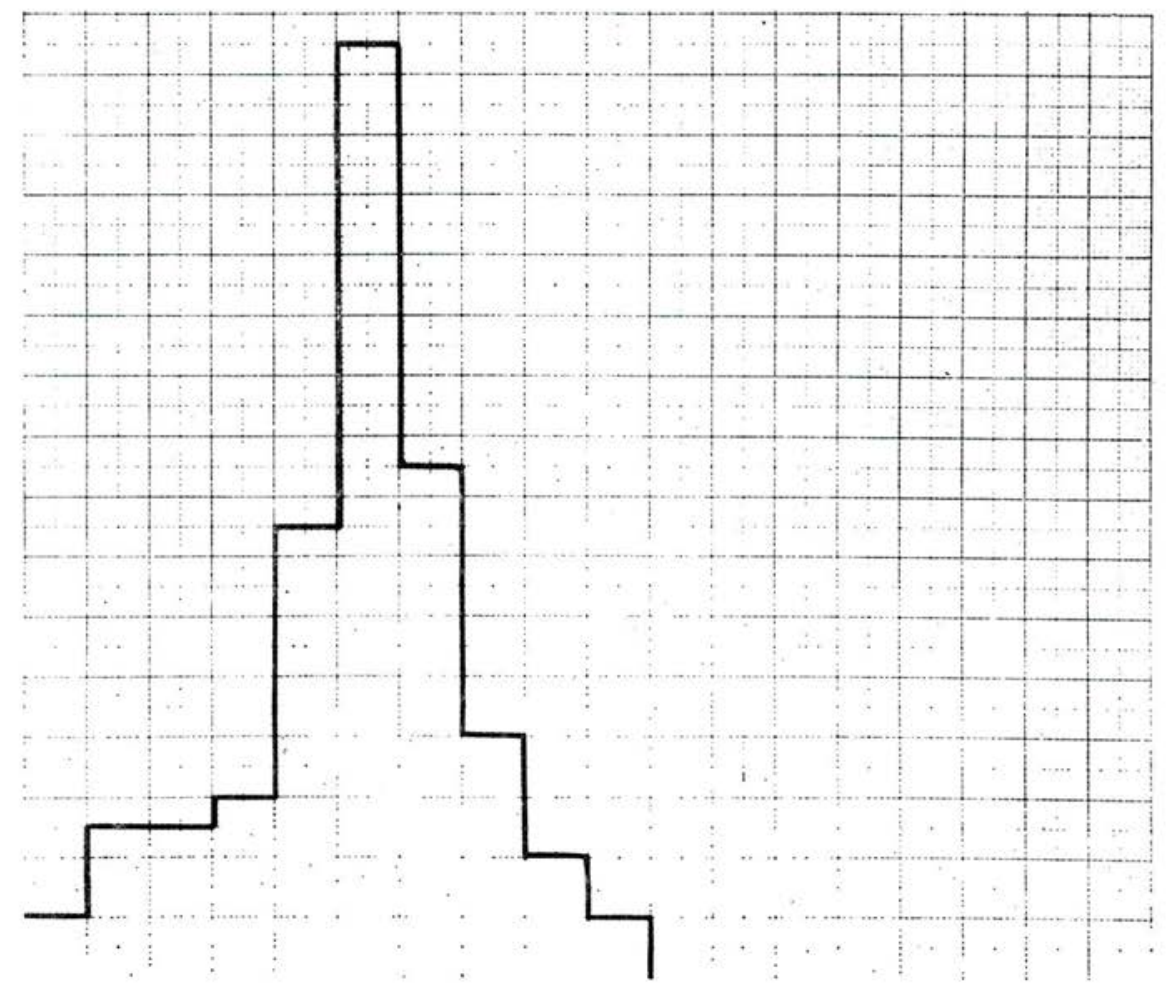

FIG. 3. - Nombre de coups par minute et par kilogramme.

Histogramme de distribution

$$
m=I_{4} \text {. }
$$

${ }^{\text {re }}$ série de mesures (Io minutes). 
Les résultats sont les suivants :

a) Examens courts (90 secondes) :

- $\bar{x}=$ moyenne $=2,33 \mathrm{CPM} / \mathrm{kg}$

- $\sigma=$ écart-type $=0,44$

- $\frac{\sigma}{\bar{x}}=$ précision $=18,8 \%$

b) Examens longs (ro minutes) :

- $\bar{x}=13,5 \mathrm{r} \mathrm{CPM} / \mathrm{kg}$

- $\sigma=1,9$

- $\frac{\sigma}{\bar{x}}=14 \%$

Les calculs sont établis sur l'hypothèse d'une distribution gaussienne. Ce choix peut se justifier par la nature des facteurs de la dispersion qui sont : la statistique radioactive et les facteurs biologiques (morphologie, corpulence, alimentation, variations physiologiques et métaboliques). Or, ces facteurs biologiques ont en général, une distribution gaussienne. L'examen des histogrammes de distribution obtenus pour cet ensemble de mesures justifie parfaitement cette hypothèse malgré le nombre relativement faible de cas.

\section{I.2. DeUXIÈME SÉRIE DE MESURES}

Cette série se compose de 100 spectres (o à $2 \mathrm{MeV}$ ) pratiqués également en 1967 dans le cadre de la surveillance systématique du personnel de laboratoire.

Ces examens sont pratiqués pendant to minutes et avec une géométrie à trois sondes.

Le spectre soigneusement examiné permet d'éliminer tout sujet " anormalement contaminé » de façon à ne conserver qu'un échantillon de population normale.

Expression des résultats et étude statistique (fig. 4)

Après mise en cartes perforées de toutes les données, les calculs ont été effectués sur ordinateur.

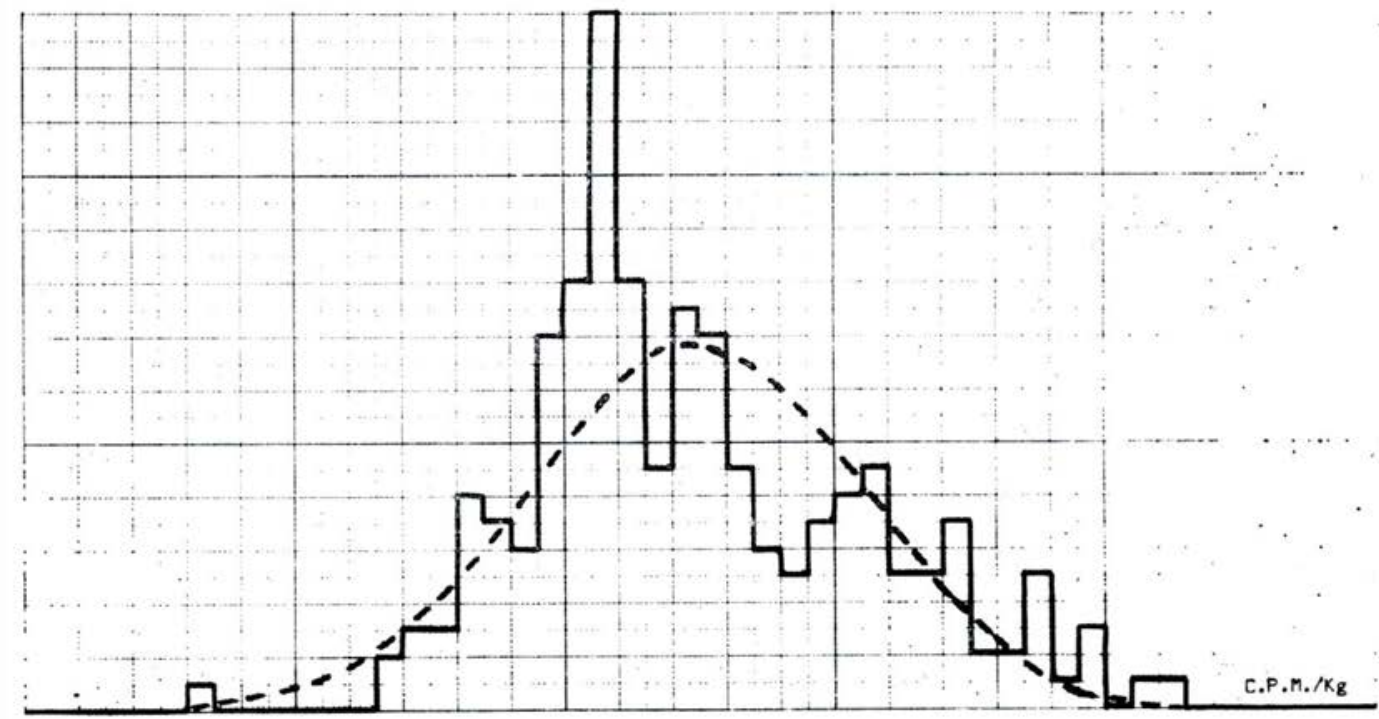

FIG. 4. - Nombre de coups par minute et par kilogramme.

Histogramme de distribution

$$
m=24,9 \text {. }
$$

$2^{\mathrm{e}}$ série de mesures (Io minutes). 
Les données programmées sont :

- le nombre total de coups en CPM/kg pour chaque individu,

- l'histogramme de distribution de cette activité,

- l'activité moyenne $\bar{x}$ en $\mathrm{CPM} / \mathrm{kg}$,

- la variance $\sigma^{2}$,

- l'écart-type $\sigma$.

Tous les résultats figurent dans des tableaux établis pour des temps de comptage allant de I à ro minutes.

Pour ro minutes, les résultats sont :

- $\bar{x}=24,9 \mathrm{CPM} / \mathrm{kg}$,

- $\sigma^{2}=38,94$,

- $\frac{\sigma}{\bar{x}}=$ précision : $25 \%$.

\subsection{Comparaison des résultats}

Ces résultats font apparaître des différences entre les deux séries de mesure.

\begin{tabular}{|c|c|c|c|}
\hline & \multicolumn{2}{|c|}{ Première série de mesure } & \multirow{2}{*}{$\begin{array}{c}\text { Deuxième série } \\
\text { 10 } \mathrm{mn}\end{array}$} \\
\hline & $90 \mathrm{~s}$ & Io $\mathrm{mn}$ & \\
\hline $\bar{x}$ en $\mathrm{CPM} / \mathrm{kg}$ & 2,33 & 13,51 & 24,9 \\
\hline$\sigma^{2}$ & 0,44 & 3,60 & 38,94 \\
\hline$\sigma$ & 0,66 & 1,9 & 6,24 \\
\hline$\frac{\sigma}{\bar{x}}$ & $18,8 \%$ & $14 \%$ & $25 \%$ \\
\hline $\begin{array}{l}\text { bruit de fond } \\
\text { moyen }\end{array}$ & I 542 pour $90 \mathrm{~s}$ & 27000 pour ro $\mathrm{mn}$ & 36000 pour $10 \mathrm{mn}$ \\
\hline poids moyen & $69,5 \mathrm{~kg}$ & $695 \mathrm{~kg}$ & \\
\hline $\begin{array}{c}\mathrm{N} \text { moyen } \\
\text { par individu }\end{array}$ & 250 coups & 9450 coups & I7 400 coups \\
\hline
\end{tabular}

Ces différences sont difficiles à expliquer car les matériels qui équipent ces diverses installations sont semblables.

On ne peut que proposer les explications suivantes :

Les examens de la deuxième série s'échelonnent sur quelques mois, tandis que ceux de la première sont groupés sur quelques semaines. Or, la contamination des populations baisse très rapidement en ce moment comme le montrent les travaux récents [IO, I I, I 2]. Ceci peut expliquer que l'écart-type est plus grand pour des mesures plus étalées dans le temps. Les mesures de la deuxième série sont antérieures à celles de la première, ce qui expliquerait que la moyenne soit plus grande dans ce cas.

Enfin, les effectifs de la première série étaient plus homogènes par leur mode de vie, leur alimentation, leur âge et leur poids. 


\section{I.4. VALIDITÉ D'Un tempS DE COMPtAge COURT}

L'adaptation de méthodes de comptages courts pose la question de la précision obtenue et de la validité des résultats. La dispersion due à la statistique de comptage est liée au temps de comptage et diminue quand il augmente tandis que la dispersion due aux facteurs individuels n'est pas liée au temps de comptage. Il importe donc de chiffrer leur part respective dans la dispersion globale obtenue expérimentalement.

I.4.I. Erreur sur un comptage de grandeur définie.

Si l'on mesure l'activité $\gamma$ d'une source, le résultat $R$ de la mesure est obtenu par soustraction du bruit de fond $B$ du comptage global $G$.

$$
R=G-B .
$$

Or, $G$ et $B$ sont dispersés autour d'une moyenne et admettent pour écart-type :

d'où

$$
\begin{gathered}
\sigma_{G}=\sqrt{G} \text { et } \sigma_{B}=\sqrt{B} \\
\sigma^{2}{ }_{R}=\sigma^{2} G+\sigma^{2}{ }_{B} .
\end{gathered}
$$

Le bruit de fond $B$ peut être déterminé avec plus ou moins de précision. Dans le cas le plus défavorable, il est déterminé par un seul comptage de même durée que la mesure. Le hasard peut faire qu'il soit très éloigné de la vraie moyenne $m$ du bruit de fond. Dans ce cas, l'erreur sur la mesure est maximale.

$\mathrm{Si}$ au contraire, $B$ est déterminé par un nombre important de mesures ou pendant un temps très long, la valeur obtenue pour $B$ peut se rapprocher autant qu'on veut de la vraie moyenne $m$, jusqu'à ce que l'erreur pour cette moyenne soit négligeable. Dans ces conditions, l'erreur maximale possible par soustraction sera la moitié de ce qu'elle est dans le cas précédent.

C'est la méthode adoptée dans les deux séries de comptage, ce qui donne :

$$
\sigma^{2}{ }_{R}=\sigma^{2}{ }^{2} \text {. }
$$

Cas du comptage long :

Soit, pour la première série de mesures :

$N=9450$, le nombre de coups moyen pour un homme de $69,5 \mathrm{~kg}$ en ro minutes.

$$
\begin{gathered}
B \text { moyen }=27000 \\
\sigma_{R}=\sqrt{27000+9450}=190 \\
\frac{\sigma_{R}}{N}=\frac{190}{9450}=2 \% .
\end{gathered}
$$

Pour la deuxième série de mesures, le même calcul donnerait : $1,3 \%$.

Cas du comptage court :

$N=250$ coups pour 90 secondes

$B$ moyen $=1550$

$$
\begin{gathered}
\sigma_{R}=\sqrt{1550+250}=42,5 \\
\frac{\sigma_{R}}{N}=17 \% .
\end{gathered}
$$

1.4.2. Calcul de l'imprécision due à la fluctuation individuelle.

Connaissant la précision statistique $\frac{\sigma_{R}}{N}$ et la précision expérimentale $\frac{\sigma}{N}$, la précision $F$ due aux fluctuation individuelles a pour expression :

$$
\mathrm{F}=\sqrt{\left(\frac{\sigma}{N}\right)^{2}-\left(\frac{\sigma_{R}}{N}\right)^{2}} .
$$


Pour les comptages longs :

$$
F_{1}=\sqrt{14^{2}-2^{2}}=\sqrt{192}=13,85 \%
$$

pour la $\mathrm{I}$ er série de mesures, et

pour la $2^{\mathrm{e}}$ série de mesures $=24,9 \%$.

Cette valeur de $F$ est obtenue en géométrie trois sondes. Pour les comptages de 90 secondes, on a utilisé la géométrie de Miller (I sonde) qui a pour avantage de réduire les fluctuations individuelles dues à la répartition anatomique des radioéléments car les divers points du corps sont vus sensiblement sous le même angle par la sonde unique.

Le même calcul dans ce cas là donne :

$$
F_{c} \simeq 8 \% \text {. }
$$

Ce résultat est bien conforme aux prévisions.

Ces calculs permettent de faire des conclusions intéressantes :

- les comptages longs sont plus précis que les comptages courts mais la différence n'est pas excessive comparée au gain de temps;

- dans les comptages longs, les fluctuations individuelles prédominent très largement sur les fluctuations statistiques de comptage;

- pour les comptages courts, les fluctuations statistiques tendent à égaler et même à dépasser les fluctuations individuelles si le taux de comptage est faible;

- la géométrie de Miller est très intéressante pour sa faible dispersion individuelle. Par contre, elle est peu sensible et demanderait un temps assez long pour donner un spectre exploitable dans le cas d'une très faible contamination.

\section{II - UTILISATION D'UN TEMPS DE COMPTAGE COURT - INDICE DE TRI}

\section{II.1. Comparaison des sujets examinés à un échantillon de référence}

Les sujets qui constituent les deux séries de mesures que nous avons exposées sont considérés comme un échantillon de population normale et servent de base de comparaison pour ces sujets examinés.

Il est évident que l'on peut faire des réserves sur le caractère normal de cet échantillon. Il parait légitime de retenir comme normal tout sujet dont le mode de vie et les activités professionnelles sont éloignés de toutes causes de contamination interne. Toutefois, dans l'éventualité d'une contamination générale et importante de la population, ce caractère de normalité serait abusif et peu conforme aux préoccupations de l'hygiène atomique; il serait alors plus logique d'établir une norme en fonction des Q.M.A.

La comparaison des sujets examinés à l'échantillon de référence repose sur les méthodes statistiques les plus élémentaires.

Soit : $\bar{x}$ le nombre moyen de CPM/ $/ \mathrm{kg}$ établi pour une installation donnée dans des conditions de comptages bien définies.

$\sigma$ l'écart-type correspondant.

Pour une telle mesure, on calcule les limites de l'intervalle de confiance $99 \%$ donné par l'expression $\bar{x} \pm 2,6 \sigma$.

Toute mesure sortant de ces limites sera considérée comme anormale. Dans le cas présent, seules les mesures sortant de la limite supérieure sont suspectes et évoquent une contamination anormale ou un incident de comptage. Par contre, une mesure inférieure à la limite basse évoque plutôt un incident de comptage.

Cette méthode implique le calcul de $x$ en CPM/kg à partir du taux de comptage $N$ et du poids $P$ du sujet.

Ce calcul peut être fait une seule fois et les résultats sont alors rassemblés dans un tableau à double entrée. 
Pour la deuxième série d'examens de longue durée, les calculs ont été programmés sur ordinateur. Les résultats sont présentés sous forme de dix tableaux correspondant à tous les temps de comptage de I minute à ro minutes. Chaque tableau indique $N$ moyen, $N$ maximum et $N$ minimum pour tous les poids de 50 à $120 \mathrm{~kg}$. Tous ces résultats ne sont pas indispensables mais ont été programmés pour profiter des possibilités offertes par l'ordinateur. Si les calculs sont faits par les moyens habituels, on se contentera de les faire pour un seul temps de comptage que l'on aura décidé d'adopter.

Les tableaux de résultats ne sont pas rapportés ici car ils sont propres à une installation bien déterminée. Tous les tableaux sont calculés à partir d'une seule série de mesures de ro $\mathrm{mn}$. Pour les temps de comptages plus courts, l'écart-type $\sigma$ augmente car la dispersion statistique de comptage augmente. Il faut en tenir compte et calculer l'écart-type propre à chaque temps de comptage. Sans l'aide d'un ordinateur ou d'un calculateur, le calcul complet est assez laborieux.

\section{A titre d'exemple :}

Pour un poids déterminé et une activité comptée moyenne égale à $\bar{N}$ :

$$
\begin{aligned}
& N_{\text {minima }}=\bar{N}-2,6 \sigma=\bar{N}(1-0,65) \\
& N_{\text {maxima }}=\bar{N}+2,6 \sigma=\bar{N}(1+0,65) .
\end{aligned}
$$

\section{II.2. INDICE DE TRI}

\section{I1.2.1. Définition.}

L'indice est le rapport entre le nombre total de coups par minute et par kilogramme mesuré pour un sujet et la valeur moyenne de CMP $/ \mathrm{kg}$ mesurée dans les mêmes conditions pour un sujet normal de même poids. Par suite de la dispersion des poids des sujets examinés, il est pratique d'exprimer les deux termes du rapport en CPM $/ \mathrm{kg}$.

Ainsi défini, l'indice de tri garde la même signification pour tous les systèmes et toutes les installations de spectrométrie. Les résultats restent comparables entre eux.

Dans ces conditions, on peut dire que tout indice dont la valeur se situe au-dessus de l'intervalle de confiance de $99 \%$, peut être considéré comme anormal et justifie un examen long.

Cette définition appelle quelques remarques :

- l'indice ne représente pas un rapport d'activité au sens strict de la définition;

- deux sujets qui ont la même charge du même radioélément et un poids très différents peuvent présenter des différences dans l'auto-absorption et par conséquent un taux de comptage différent.

Deux indices de tri semblables n'expriment une identité de charge corporelle que dans la mesure où la morphologie des sujets et la nature des radioéléments en cause sont semblables.

En effet, il peut arriver que des radioéléments différents par leur énergie, par leur localisation métabolique et leur quantité, donnent des indices voisins pour des charges très différentes.

Cependant, l'expérience a montré, sur mannequin et sur sujets contaminés que cet indice est un indicateur très sensible de contamination interne par radioéléments émetteurs $\gamma$.

\section{II.2.2. Etalonnage des installations.}

L'étalon retenu dans cette étude pour déterminer l'indice est l'individu normal défini statistiquement. Cette méthode est criticable et l'étalon ainsi défini n'est pas pratique car il est très inconstant dans le temps.

En effet, la charge moyenne en $\mathrm{Cs}^{137}$ qui était de l'ordre de $15 \cdot 10^{-9} \mathrm{Ci}$ en 1965 , est actuellement en France de l'ordre de 3 à $4 \cdot 10^{-9} \mathrm{Ci}[10,11,12]$. Le taux global de comptage sur ro minutes est tombé dans le même temps en Métropole et avec notre installation de 30000 coups à 10000 .

Les inconvénients sont évidents, il faut redéfinir à intervalles rapprochés la notion d'individu normal, ce qui nécessite un échantillonnage et des calculs laborieux.

Il est plus conforme à la notion d'étalon de définir un étalon conventionnel stable.

Nous avons adopté à cet effet l'individu moyen normal au début de 1968, caractérisé par un mannequin équivalent [13]. Ce mannequin grossièrement anthropomorphe est en polyéthylène (tissu équivalent). Il contient $140 \mathrm{~g}$ de potassium et $\mathrm{s} \mathrm{nCi}$ de $\mathrm{Cs}^{137}$, répartis uniformément. 
Les caractéristiques sont les suivantes :

- Poids total : $70 \mathrm{~kg}$

- Masse liquide : $56 \mathrm{~kg}$.

- Répartition :

\begin{tabular}{|c|c|c|c|}
\hline & $\begin{array}{l}\text { Parties molles } \\
(\% / \%)\end{array}$ & $\begin{array}{c}\mathrm{Os} \\
(\% / o o)\end{array}$ & $\begin{array}{l}\text { Total } \\
(\% / o o)\end{array}$ \\
\hline tête & 54 & 8 & 62 \\
\hline cou & 8 & 2 & 10 \\
\hline membres supérieurs & 56 & 6 & I 24 \\
\hline cuisses & IOI & 9 & Iro \\
\hline jambes & 29 & 6 & 70 \\
\hline \multirow[t]{2}{*}{ tronc } & 490 & 24 & 514 \\
\hline & & Total : & I 000 \\
\hline
\end{tabular}

Une série de mesures faites au début de 1968 donne un spectre du mannequin absolument superposable à celui de l'homme moyen de $70 \mathrm{~kg}$.

Un tel étalon est envoyé périodiquement aux différentes installations de spectrométrie humaine. Ainsi, les indices de tri obtenus dans chacune des installations ont la même signification et sont comparables. Il serait possible de réaliser également un mannequin contenant un multiple entier de la charge normale. Cette méthode permet des mesures plus précises et diminue les fluctuations de comptage. Il suffit de diviser les résultats obtenus par le multiple adopté ce qui ramène à l'étalon normal.

L'utilisation d'un tel étalon ne dispense pas, bien entendu, de faire régulièrement pour chaque installation l'étude statistique complète de la distribution.

\section{III - MÉTHODE GÉNÉRALE UTILISABLE \\ POUR LA SURVEILLANCE DU PERSONNEL EXPOSÉ AUX RAYONNEMENTS}

\section{III.I. PRINCIPE DE LA MÉTHODE}

La première série de mesures a été faite pour des temps de comptage très différents 90 secondes (Miller) et ro minutes ( 3 sondes).

Dans la deuxième série de mesures les calculs ont été faits sur 10 minutes ( 3 sondes) et à partir de ces données expérimentales le calcul a été fait pour des temps allant de 10 minutes à une minute. Ces deux séries de mesures permettent de conclure dans le même sens. L'imprécision due aux fluctuations individuelles n'est pas influencée par le temps de comptage. Son importance est telle, en regard des fluctuations statistiques de comptage, que l'augmentation du temps de comptage n'améliore pas de façon appréciable la précision statistique globale. On peut donc envisager de remplacer les examens de spectrométrie humaine de routine de ro minutes par des examens de plus courte durée. Il faut donc en définir les modalités : durée de l'examen, géométrie et expression des résultats.

La géométrie et la durée de l'examen sont en réalité liées. En effet, la géométrie de Miller utilisée au début de cette étude présente un inconvénient important pour les mesures de sujets non contaminés ou très peu contaminés. Le taux de comptage est alors faible et par conséquent la dispersion sur le comptage est élevée. De plus, il est préférable de conserver la même géométrie pour toutes les mesures qu'elles soient courtes ou longues. 
Enfin, pour obtenir le maximum de renseignements à partir d'un même examen, il a paru utile d'obtenir en même temps que le résultat du comptage global, transformé en CPM/kg, puis en indice, le spectre du sujet examiné.

En effet, l'indice de tri pour une mesure de courte durée est un bon indicateur d'une contamination éventuelle. Mais il faut souligner à nouveau les réserves qui ont été faites plus baut : il ne s'agit pas d'une véritable mesure d'activité, deux sujets ayant le même indice n'ont pas forcément la même contamination, etc.

En ajoutant à l'indice de tri le spectre intégral du sujet, on apporte un argument supplémentaire de la non-contamination du sujet, et nous avons pris comme seuil à partir duquel le spectre est exploitable, le temps de comptage nécessaire pour faire apparaitre de façon significative le pic du Potassium 40.

\section{iir.2. Application pRatique}

Ces considérations nous ont amené à adopter pour l'examen de routine du personnel exposé aux rayonnements un examen de deux minutes en géométrie trois sondes. En effet, pour la première série de mesures, on a vu que la précision statistique sur le taux de comptage de ro $\mathrm{mn}$ est de $2 \%$. Pour un comptage de deux minutes, cette valeur sera multipliée par $\sqrt{5}$, elle devient : $4,48 \%$. Cette valeur reste faible devant les fluctuations individuelles et la précision statistique globale n'est pas très modifiće. Pour la première série de mesures, elle est égale, en effet, à :

$$
P=\sqrt{(13,85)^{2}+(4,48)^{2}}=14,6 \%
$$

au lieu de $14 \%$ pour un comptage de ro minutes. Cette estimation théorique est confirmée par une première série de mesures sur 2 minutes qui donne une valeur de is $\%$. table.

Une mesure de deux minutes sur trois sondes permet également d'obtenir un spectre exploi-

En pratique, nous avons donc adopté pour l'ensemble de nos installations la mesure de deux minutes trois sondes. Une échelle de comptage branchée sur le sélecteur permet de voir au bout de deux minutes si l'indice est normal.

Dans ce cas, le spectre de l'individu est enregistré. Si l'examen de ce spectre ne montre aucune anomalie, l'examen est terminé.

Dans tous les autres cas, indice trop bas (incident de comptage) ou indice trop élevé (contamination probable), anomalie du spectre, l'examen est poursuivi pendant 8 minutes.

Dans ces conditions, il n'y a aucune parte de temps puisque le spectre peut être enregistré sur imprimante pendant que le sujet sort de l'enceinte et qu'un nouveau sujet prend sa place. Au total, le débit de l'installation passe pour des examens normaux à is sujets à l'heure au lieu de 4 à l'heure pour les examens de 10 minutes.

\section{III.3. Discussion}

\section{Valeur de la métbode :}

L'exploitation de l'indice de tri nécessite la définition de valeurs limites considérées comme normales. Ces valeurs doivent être fixées pour chaque groupe d'installation en fonction de la distribution de la population, et de son écart-type et également en fonction du risque particulier de la population considérée. En effet, il est nécessaire de calculer les variations de cet indice en fonction du taux de contamination par le ou les radioéléments considérés. Dans ces conditions, nous avons fixé les valeurs normales de l'indice de tri comme étant comprises entre 0,7 et 1,3 . Si on tient compte de l'intervalle de confiance de $90 \%$ et dans l'hypothèse la plus défavorable d'une contamination interne par un mélange de Produits de Fission (émetteurs $\gamma$ ), on risquerait de négliger avec ces valeurs, une dose d'irradiation interne quasi nulle de $2,25 \mathrm{mrem} / \mathrm{an}$. Ce calcul est, bien entendu, très approximatif mais donne un ordre de grandeur.

Naturellement, depuis le début de cette étude, la distribution de la population examinée a été régulièrement contrôlée.

\section{Facteur de pondération.}

On peut faire des réserves sur la valeur du poids qui est choisi ici comme facteur de pondération. Le poids en effet, ne reflète pas exactement la morphologie du sujet examiné. Il semblerait plus logique d'adopter comme Marinelli [14] le facteur épaisseur soit $\sqrt{\frac{\text { poids }}{\text { hauteur }}}$. Dans le 
cadre d'une étude en cours sur le traitement de l'information radiobiologique [is], nous notons maintenant systématiquement, parmi d'autres données, la taille du sujet et nous pensons améliorer la précision de cette étude en utilisant le facteur proposé par MARINELLI. Son intérêt est d'atténuer les fluctuations dues à la morphologie du sujet qui sont sensibles dans notre géométrie trois sondes.

\section{CONCLUSION}

L'examen de spectrométrie humaine de deux minutes, appliquée à la détection des émetteurs $\gamma$ d'énergie supérieure à $200 \mathrm{keV}$ en géométrie " trois sondes " est suffisamment précis pour les besoins de la surveillance systématique du personnel exposé aux rayonnements.

En effet, l'examen de deux minutes trois sondes, présente plusieurs avantages :

- il permet d'accroitre nettement le débit de fonctionnement d'une installation de spectrométrie humaine;

- sa précision est bonne, comparativement à un examen de ro minutes;

- il utilise la même géométrie que l'examen de longue durée et permet de passer de l'un à l'autre sans perte de temps,

- il permet d'effectuer un tri à partir d'un indice dont les limites sont telles qu'on est certain de ne pas négliger une contamination interne par émetteurs $\gamma$;

- le diagnostic de non-contamination peut être enfin confirmé par le spectre du sujet.

\section{BIBLIOGRAPHIE}

[i] Frossard H., Arnaud G., Andrieu L., Fabre J., Dongradi G. Etude d'une installation légère en spectrométrie gamma humaine. Radioprotection, Dunod, 1968, vol. $3, \mathrm{n}^{\circ} 4$.

[2] Directory of whole-body radioactivity monitors, A.I.E.A., Vienne 1964 .

[3] Radioactivity in Man. Second Symposium. Charles C. Thomas, Publisher, 1965.

[4] Radioactivity in Man. Symposium. Charles C. Thomas, Publisher 196r.

[s] Whole-body counting. Symposium de Vienne, A.I.E.A., 13-16 juin r96r.

[6] Assessment of Radioactivity in man, Vol. I et 2. Symposium Heidelberg, A.I.E.A., I I-16 mai, 1964 .

[7] Dr. Smeets J. et Dr. Sclunier H. Résultats de l'étude comparative internationale des mesures effectuées dans la communauté européenne à l'aide d'anthropogammamètre. Conférence de l'Association "italiana di Fisica sanitaria e protezione contro le radiazoni", 29-3 I mai 1968.

[8] Anderson E.C. - U.C.L.A. Organic scintillation detectors and their use in the study of body composition. Public conference on body composition, 4-6 mai 1967.

[9] Measurements of gamma ray in vivo. Rapport ANL. 5596.

[10] Godfrey B.E., Vemart J. Measurements of Cesium 137 in buman beings in 1958 - 1967. Nature vol. 218, May 25, 1968.

[i1] Garnier A. Etude bibliographique des paramètres radiobiologiques du Césium $\mathrm{I} 37$ nécessaires à l'éraluation des doses d'irradiation interne en fonction de l'âge. Rapport CEA, R-3432, 1968.

[12] Fabre J., Andrieu L., Arnaud G., Frossard H., Bock B. Evolution de la charge du corps humain en Césium 137 dans la région parisienne de 1964 à 1967 . Radioprotection. Dunod 1968, Vol. 3, $\mathrm{n}^{\circ}$ 3, pp. 173-183.

[13] Fabre J., Flauder M., Frossard H., Andrieu L. Mannequin à formes géométriques simples utilisable en spectrométrie bumaine. Rapport $\mathrm{n}^{\circ}$ 9/CRSSA/HA, 1967. Centre de Recherches du Service de Santé des Armées.

[14] Marinelli L.D. ANL 7220, July 1965. Geometrical and Physical parameters in wholebody Gamma ray spectrometry measurements.

[is] Frossard H., Rocquet G., Morin D., Arnaud G., Golhen A. Informatique appliquée à la surveillance médico-radiobiologique. Rapport no $5 / C R S S A / H A, 26-11-1968$. Centre de Recherches du Service de Santé des Armées. 